\title{
On the Identification Technology for the Random Load Spectrums
}

\author{
W.M. Li, H.D. Wang \\ Shanghai Spaceflight Precision Machinery Research \\ Institute \\ Shanghai, China
}

\author{
Y.Y. Qiu, Q. Niu \\ School of Mechano-Electronic Engineering \\ Xidian University \\ Xi'an, China
}

\begin{abstract}
This paper deduces the relationship between the frequency response and loads for a structure within the frequency domain, and puts forward a new method for identifying the random loads spectrum. Firstly, the response power spectrum of structural control points is calculated based on the white noise loads spectrum. Then in order to get the corresponding random loads spectrum, the response power spectrum and the desired output response spectrum are compared. Finally, a specific cabin structure is analyzed by using the above method. The FEA (Finite Element Analysis) simulation results show that the method is valid in identifying the fundamental acceleration loads spectrum.
\end{abstract}

Keywords-frequency response; random load spectrum; response power spectrum; FEA

\section{INTRODUCTION}

In the industrial community, the complexities of vibration environments often restrict the direct measurements to the external loads of specific objects; for example, it is difficult to measure the dynamic loads generated when a aircraft is flying, also the dynamic loads created when an earthquake or storm happen. As a result, the methods about loads identification have attracted much attention. In a word, technicists can derive the dynamic loads using the load identification methods after evaluating the dynamic response of target bodies and analyzing its dynamic property, which supports the machine design and analysis[1].

There are two ways for the identification of the vibration loads: frequency domain method and time domain method. The time domain method is not widely recognized since the complexities of random process and huge computation. The frequency domain method defines the models referring to the relationship between system inputs and outputs within frequency domain and identifies the dynamic inputs based on the outputs. Because of the linearity of model within the frequency domain such that the reverse calculation is easy to handle with, researchers have made great progress in the load recognition. Mira et al. [2] identified the impulse loads with the mini-wave method using FEA; and discussed how to set the boundary condition in mini-wave recognition. M.C. Djamaa et al. [3] had further understood about the dynamic loads distribution of the thin pilaster structure using the finite differential method; and eliminated the error amplification through normalized. Choi et al. [4,5] improved the stability of the load identification results and studied the affect on loads identification accuracy selecting different normalization parameter. In order to identify a two-dimensional dynamic loads distribution, Paper [6] utilized the tensor theory to generate the high-dimensional moment basis function in the high dimensional orthogonal space and then represented the unknown two-dimensional dynamic load distribution with the undetermined coefficient in the form of high dimensional orthogonal function series. In order to identify the dynamic load more accurate and stable, Paper [7] developed two new regular methods based on the singular value theory of compact operate. Paper [8] put forward the condition number weighted algorithm based on inverse pseudo-excitation method, which improves the error amplifier problems of the identification results generated when matrix is "sick" in random loads identification. The methods offered in [2-8] solve the problem of identifying the dynamic loads mathematically, but they were all limited to be academic because of the complexities of the theory and the difficulty of large amount of calculation.

In this paper, a new random loads identification method, based on the relationship between random vibration responses and loads within the frequency domain, is investigated. Besides, an example proves its effectiveness by inversely testing the fundamental acceleration load spectrum for a specific cabin structure.

\section{THE RANDOM LOAD IDENTIFICATION ALGORITHM}

\section{A. Assumptions}

Normally, people make assumptions to define the relationship between system inputs (Unknown load spectrum) and outputs (Control spectrum)[7]. For the analysis, it is assumed that:

1) The target system is linearly time-invariant. load.

2) The target system is impacted by the stable random

3) The location of the random loads is known and fixed.

4) It is only the unknown random loads that affect the entire system response.

\section{B. Mathematical Model}

To a linear system suffered the random stable loads, the relationship between the loads and the response can be analyzed within both the frequency and the time domain. In the time domain, the system impulse response function $h(t)$ 
connects the load $x(t)$ with response $y(t)$; In the frequency domain, it is the system frequency response function $H(f)$ that bridges from the load $X(f)$ to the response $Y(f)$. The transfer function relationship is depicted in Fig. 1 as below.

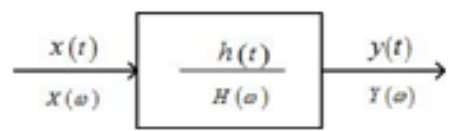

FIGURE I. LOAD AND RESPONSE OF THE SYSTEM.

In the time domain, the response $y(t)$ is written as:

$$
y(t)=\int_{-\infty}^{\infty} h(\tau) x(t-\tau) d \tau
$$

Perform the Fourier Transform to both sides of the equation (1). From the theory of convolution, Eq. (1) can be transferred into

$$
Y(f)=H(f) X(f)
$$

Eqs. (1) and (2) describe the relationship between the system input (The load), the system response transfer function (Dynamic characteristics) and the system response (Output).

After performing the Fourier Transform, the system frequency response transfer function is

$$
H(f)=\int_{-\infty}^{\infty} h(\tau) e^{-i 2 \pi f \tau} d \tau
$$

So that, the self-correlation function of the system response becomes:

$$
\begin{aligned}
R_{y}(\tau) & =E[y(t) y(t+\tau)] \\
& =\int_{-\infty}^{\infty} \int_{-\infty}^{\infty} h\left(\tau_{1}\right) h\left(\tau_{2}\right) R_{x}\left(\tau+\tau_{1}-\tau_{2}\right) d \tau_{1} d \tau_{2}
\end{aligned}
$$

In the Eq. (4), $R_{x}\left(\tau+\tau_{1}-\tau_{2}\right)$ is the self-correlation function of the load.

Again, after performing the Fourier Transformation to both sides of Eq. (4), the equation shows how the response power spectrum works with the load power spectrum and can be written as:

$$
\begin{aligned}
S_{y}(f) & =\frac{1}{2 \pi} \int_{-\infty}^{\infty} R_{y}(\tau) e^{-i 2 \pi f \tau} d \tau \\
& =|H(f)|^{2} S_{x}(f)
\end{aligned}
$$

In Eq. (5), $H(f)$ is a complex number which contains the magnitude and phase of the system. But, $|H(f)|^{2}$ changes to be a real number holding the magnitude only. And Eq. (5) shows that the response power spectrum is equal to the product of the square of the system frequency response function $\left(|H(f)|^{2}\right)$ and the load power spectrum $\left(S_{x}(f)\right)$.

At first, assume that the input to the system is a white noise. Then the acceleration response power spectrum of the system can be derived from Eq. (5) as

$$
\tilde{S}_{y}(f)=|H(f)|^{2}
$$

As $\hat{S}_{y}(f)$ represents the acceleration response power spectrum of the control system, the necessary fundamental acceleration load spectrum $\hat{S}_{x}(f)$ for the system is

$$
\hat{S}_{x}(f)=\frac{\hat{S}_{y}(f)}{|H(f)|^{2}}=\frac{\hat{S}_{y}(f)}{\tilde{S}_{y}(f)}
$$

The reverse derivation of the fundamental acceleration load spectrum is shown as above. The accuracy of the load identification could be tested directly: at first, exciting the system using the fundamental acceleration load spectrum shown in Eq. (7) as the input; then calculating the acceleration response power spectrum of the control points $S_{y}(f)$ and compare it with the control acceleration response power spectrum $\hat{S}_{y}(f)$. Finally obtaining the identification accuracy in the error form.

$$
\text { error }=\frac{\left|S_{y}(f)-\hat{S}_{y}(f)\right|}{\hat{S}_{y}(f)} \times 100 \%
$$

\section{SIMULATION RESULT}

In this section, the simulation is conducted for a specific cabin structure to prove the effectiveness of the new method. In Fig. 2, the cabin structure consists of the enclosure and internal side plates.

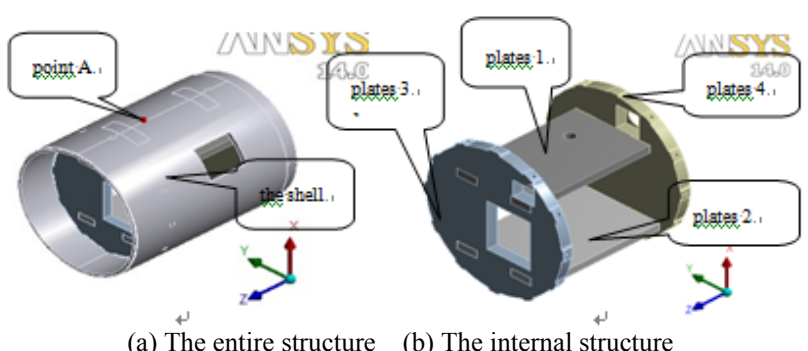

(a) The entire structure (b) The internal structure

FIGURE II. FIGURE 2: THE TYPICAL CABIN STRUCTURE.

The material for the cabin structure is chosen to be the LY12CZ aluminum alloy and the technical specifications are listed as: the elasticity modulus $\mathrm{E}=2.1 \mathrm{e} 11 \mathrm{~Pa}$; the Poisson's ratio $\mu=0.3$; the density $\rho=2700 \mathrm{Kg} / \mathrm{m}^{3}$. To realize the random vibration of the cabin structure, four surfaces are fixed completely shown in Fig.3.

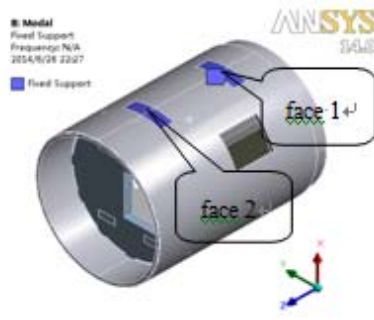
$\begin{array}{ll}\text { (a) The fixed constraints } 1 \text { and } 2 & \text { (b) The fixed constraints } 3 \text { and } 4 \\ \text { FIGURE III. THE CONSTRAINTS FOR THE TYPICAL CABIN }\end{array}$ STRUCTURE.

Assume that the fundamental acceleration load spectrum in the $X$ direction is the main stimulation acting on the four surfaces. In detail, the power spectrum density of the 
acceleration response in the $X$ direction at the point $\mathrm{A}\left(\hat{S}_{\text {out }}^{A}(f)\right)$ is a given trapezoidal spectrum shown in Fig.4.

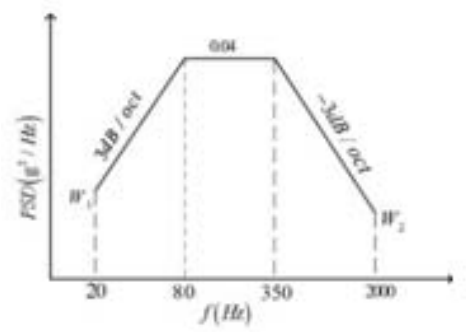

FIGURE IV. THE POWER SPECTRUM OF CONTROL OUTPUT ACCELERATION

As shown in Fig. 4, the double logarithm coordinate is utilized. Besides, the slope "N" by definition can be expressed as $d B /$ oct , which means the unit octave (oct) increases or decreases the power spectrum density in " $d B$ ". The slope is definded as:

$$
N=\frac{d B}{o c t}=\frac{10 \lg \frac{\mathrm{W}_{H}}{\mathrm{~W}_{L}}}{\log \frac{f_{H}}{f_{L}}}=\frac{10 \lg \frac{\mathrm{W}_{H}}{\mathrm{~W}_{L}}}{\lg \frac{f_{H}}{f_{L}}} \lg 2
$$

Where $f_{H}$ is the highest frequency $(\mathrm{Hz}) ; f_{L}$ is the lowest frequency $(\mathrm{Hz}) ; W_{H}$ is the power spectrum density when the frequency is $f_{H}\left(g^{2} / \mathrm{Hz}\right) ; W_{L}$ is the power spectrum density when the frequency is $f_{L}\left(g^{2} / \mathrm{Hz}\right)$.

It is easy to get $W_{1}=0.01 \mathrm{~g}^{2} / \mathrm{Hz} 、 W_{2}=0.007 \mathrm{~g}^{2} / \mathrm{Hz}$ using Eq. (9). Then, get that the power spectrum of control acceleration $\hat{S}_{\text {out }}^{A}(f)$ is

$$
\hat{S}_{\text {out }}^{\wedge}(f)=\left\{\begin{array}{lr}
10^{(-2+0.9966 \lg 0.05 f)} & (20 \mathrm{~Hz} \leq f<80 \mathrm{~Hz}) \\
0.04 & (80 \mathrm{~Hz} \leq f<350 \mathrm{~Hz}) \\
10^{(-1.3979-0.99661 \mathrm{lg} 0.0028575 f)} & (350 \mathrm{~Hz} \leq f<2000 \mathrm{~Hz})
\end{array}\right.
$$

At this time, imposing a white noise input whose amplitude is 1 and bandwidth ranges from $20 \mathrm{~Hz}$ to $2000 \mathrm{~Hz}$ to the constraint surfaces 1 to 4 and then performing the random vibration simulation, the acceleration response power spectrum for point $\mathrm{A} \tilde{S}_{\text {out }}^{\wedge}(f)$ is shown in Fig.5.

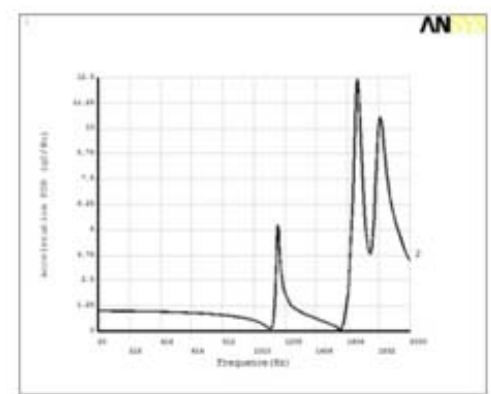

FIGURE V. ACCELERATION RESPONSE POWER SPECTRUM FOR POINT A.

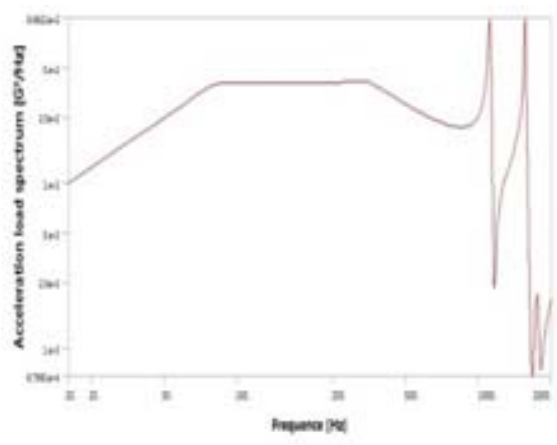

FIGURE VI. THE AVAILABLE FUNDAMENTAL ACCELERATION LOAD

Since the $\hat{S}^{A}(f)$ is assumed to be a trapezoidal spectrum expressed in Eq. (10), the corresponding fundamental acceleration load spectrum $\hat{S}_{\text {in }}(f)$ for the constraint surfaces 1 to 4 is also available after reverse derivation in application of Eq. (11). The result is shown in Fig. 6 as follow

$$
\hat{S}_{\text {in }}(f)=\frac{\hat{S}_{\text {out }}^{A}(f)}{|H(f)|^{2}}=\frac{\hat{S}_{\text {out }}^{A}(f)}{\tilde{S}_{\text {out }}^{A}(f)}
$$

Then to test the accuracy of the method, the best way is to apply the derived undamental acceleration load spectrum shown in Fig. 6 to the constraint surfaces. Comparing the resulted output response spectrum and the one given before and referring to Fig. 7, the simulation results match really well with the desired output, which indirectly show that the new method is valid.

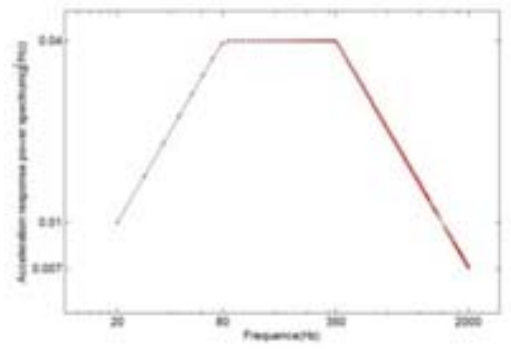

FIGURE VII.

IDENTIFIED ACCELERATION LOAD SPECTRUM AND THE CONTROL PECTRUM

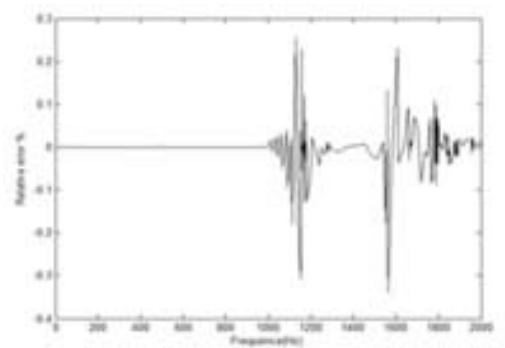

FIGURE VIII. ERROR OF THE ACCELERATION RESPONSE POWER SPECTRUM.

Fig. 8 elaborates the error distribution calculated using Eq. (8) and it is clear to see that the output error appears in the proximities of some principal resonant frequency points. 
However, a maximum only $0.3385 \%$ is achieved, which is too small to be ignored.

\section{CONCLUSION}

The article comes up with a convenient random load identification method based on the relationship between the random vibration load and corresponding response in the frequency domain. In order to get the corresponding random load spectrum, the response power spectrum under the white noise load spectrum and the desired output response spectrum are compared. According to the typical cabin structure model, we modeled the power spectrum of acceleration response of the control points on the cabin as an trapezoidal spectrum and applied the method to recognize the fundamental acceleration load spectrum on the constraint surfaces of the cabin structure. After comparing the control point acceleration response power spectrum under the recognized load spectrum with the control spectrum we obtained the maximum error to be $0.3385 \%$, which suggests high precision and also proves the accuracy and feasibility of the method.

\section{REFERENCE}

[1] Y.Y. Hu, Z.J. S, W.Y. Li, Study on Machine's Load Identification Technique. Noise and Vibration Control, (4), pp.1-5, 2011.

[2] Mita M, Gopalakrishnan S., Spectrally formulated wavelet finite element for wavepropagation and impact force identification in connected 1-D waveguides. International Journal of Solids and Structures, 42(16-17), pp.4695-4721, 2005.

[3] Djamaa M.C, Ouelaa N, Reconstruction of a distributed force applied on a thin cylindricalshell by an inverse method and spatial filtering. Journal of Sound and Vibration, 301(3-5),pp. 560-575, 2007.

[4] Choi H G, Thite A N, Thompson D J. A threshold for the use of Tikhonov regularization in inverse force determination. Applied Acoustics, 67(7), pp. 700-719, 2006.

[5] Choi H G, Thite A N, Thompson D J. Comparison of methods for parameter selection in Tikhonov regularization with application to inverse force determination. Journal of Sound and Vibration, 304(3-5), pp.894-917, 2007.

[6] Y.T. Qin, G.P. Cheng, F. Zhang. Moment method of two-dimension distribution load identification. Journal of Vibration and Diagnosis, 32(1), pp. 34-41, 2012.

[7] J. Liu. Research on computational inverse techniques in dynamic load identification. Hunan University,2011.

[8] J. Liao . Research on respones analysis of structure random vibration and random loading identification based on orthogonal decomposition. Harbin Institute of Technolog University, 2011. 